\title{
Role of vascular endothelial growth factor receptor in the pro-proliferation activity of CD40-CD40L in AGS gastric cancer cells
}

\author{
Yongling Ning, ${ }^{\mathrm{a}, \mathrm{b}}$, Keqing Qian ${ }^{\mathrm{a}}$, Chunjian Qi ${ }^{\mathrm{a}, \mathrm{c}}$ \\ ${ }^{a}$ Department of Oncology, ${ }^{b}$ Central Laboratory, The Affiliated Hospital of Nanjing Medical University, \\ Changzhou No.2 People's Hospital, Changzhou 213003, China. 'Tumor Immunobiology Program, \\ James Graham Brown Cancer Center, University of Louisville, Louisville, KY 40202, USA
}

\begin{abstract}
Background: CD40 is a type $\alpha$-membrane protein of the tumor necrosis factor receptor super-family, and CD40induced responses may mediate growth and angiogenesis in carcinoma cells.

Objectives: Define the effect of CD40 ligation on AGS gastric cancer cell line and the role of vascular endothelial growth factor/vascular endothelial growth factor receptor (VEGF/VEGFR) signals in this process.

Methods: We treated AGS cells with $1 \mu \mathrm{g} / \mathrm{mL}$ soluble CD40 ligand (sCD40L) with or without pre-incubation of either anti-VEGF mAb (MAB293) or VEGFR tyrosine kinase inhibitor (SU5416). We determined the growth effects by cell counts or $\left[{ }^{3} \mathrm{H}\right]$-thymidine incorporation assay and VEGF levels in cell-free supernatant using enzymelinked immunosorbent assays.

Results: The engagement of CD40-induced AGS cells proliferation accompanied by a significant increase autocrine VEGF through PI3K activation ( $\mathrm{p}<0.05$ ), and exogenous VEGF alone had no effect on spontaneous cell growth. SU5416 with a concentration of $8 \mu \mathrm{M}$ lead to a dramatic decrease in cell survival induced by sCD40L $(\mathrm{p}<0.05)$, whereas MAB293 did not have the similar effect $(\mathrm{p}>0.05)$.

Conclusion: CD40-CD40L interaction promoted AGS cancer cell line proliferation through a VEGFR-dependent signal pathway in the presence of an internal autocrine loop.
\end{abstract}

Keywords: CD40, gastric cancer, sCD40L, VEGF, VEGFR

Gastric cancer (GC) is one of the most common cancers worldwide. Despite the efforts and advances in the diagnosis and treatment, GC remains the second leading cause of cancer mortality [1]. It was suggested that the tumor micro environment played a prominent role in clonal expansion of gastric cancer cells, which progressively accumulated in vivo, whereas it rapidly underwent apoptosis when cultures in vitro [2]. An increasing number of molecules had been found to associate with gastric cancer cells [3].

Correspondence to: Dr. Chunjian Qi, Department of Oncology, the Affiliated Hospital of Nanjing Medical University, Changzhou No. 2 People's Hospital, Changzhou 213003, P.R. China. E-mail: qichunjian@yahoo.com.cn; c0qi0001@louisville.edu
CD40, a type membrane protein of the tumor necrosis factor receptor (TNFR) super-family, was expressed on a variety of normal cells such as B cells, macrophages, dendritic cells, and epithelial and endothelial cells. CD40 ligand (CD40L, also called CD154), a type integral membrane protein, was mainly expressed in activated T cells, B cells, and platelets. The interaction between CD40 and CD40L was crucial for both innate and adaptive immune responses [4, 5]. Previous studies showed that the activation of CD40 signal lead to B lymphocytes proliferation, germinal center formation, isotype switching and memory B cells induction [6]. Furthermore, the genetic mutations of CD40L could make the protein incapable of engagement with CD40 resulting in the defection of T cell function and cell-mediated immunity [7]. 
Moreover, CD40 was found on a wide range of tumor cells, including malignant hemopoietic cells like multiple myeloma (MM), lymphoma, and leukemia, and solid tumors like liver, bladder, breast, and gastric cancers [8]. Similarly, CD40-induced responses mediate growth and angiogenesis in carcinoma cells $[9,10]$. Thus, CD40 expression might serve as a prognostic marker in lung cancer, which correlated with metastatic spread and poor prognosis [11]. Those results imply that the CD40 pathway is promisingly exploited for cancer therapy. Some investigators reported that AGS gastric cancer cell line had highlevel expression of CD40 [12, 13]. In the present study, we investigated the effect of CD40 stimulated by sCD40L on AGS gastric cancer cell line and the mechanism, and demonstrated that VEGFR was essential for SCD40L-induced proliferation.

\section{Materials and methods Materials}

AGS human gastric cancer cells were obtained from the American Type Culture Collection. Ham's F12 medium and fetal bovine serum (FBS) were from Invitrogen (Rockville, USA). The phosphoinositide 3kinase (PI3K) inhibitor LY294002 and vascular endothelial growth factor receptor (VEGFR) tyrosine kinase inhibitor SU5416 were purchased from Calbiochem. Human vascular endothelial growth factor (VEGF) Sandwich enzyme-linked immunosorbent assays (ELISA) kit, anti-human VEGF neutralizing monoclonal antibody (mAb) (MAB293), recombinant human VEGF were all from R\&D Systems (Minneapolis, USA). $\left[{ }^{3} \mathrm{H}\right]$-thymidine was from GE Healthcare Bio-Sciences (Piscataway, USA).

\section{Cell line culture}

AGS, a CD40 positive gastric cell line free of mycoplasma contamination, was maintained in F12 medium supplemented with $10 \%(v / v)$ FBS at $37^{\circ} \mathrm{C}$ in a humidified $5 \% \mathrm{CO}_{2}$ atmosphere incubator (Jouan, Saint-Herblain, France). The culture media was changed 24 hours before each experiment, and AGS cell line was incubated with sCD40L for 24 hours or indicated time when cells confluence was $80 \%$. The cell-free supernatants were collected, and the levels of VEGF were measured according to manufacture's guidelines.

\section{[ $\left.{ }^{3} \mathrm{H}\right]$-thymidine incorporation assay $\left.\left({ }^{3} \mathrm{H}\right]-\mathrm{TdR}\right)$}

The proliferation was measured by the assay of $\left[{ }^{3} \mathrm{H}\right]-\mathrm{TdR}$ as described previously [14]. Cells were inoculated in triplicate at $4 \times 10^{4}$ cells/well (96-well plate) (96-well plate) in $100 \mu \mathrm{L}$ of F12 (10\% FBS) with the indicated treatments. [3H]-thymidine $(1 \mu \mathrm{Ci})$ was added and incubated for the last 16 hours. Then, cells were counted in a liquid scintillation counter (Beckman-Coulter, Fullerton, USA) using the following:

$$
\mathrm{SI}=\frac{\text { Experimental group (cpm) - Backgroup (cpm) }}{\text { Control (cpm) - Backgroup (cpm) }}
$$

\section{Statistical analysis}

The statistical analysis of the data was performed using SPSS 13.0 software, comparing the means between two groups using the Student's t-test.

\section{Results \\ CD40 ligation obviously profited the growth of AGS cells}

To assess the potential proliferation effect of CD40L in activation of CD40 signaling, we examined the expression of CD40 in AGS cells and treated the cells with the sCD40L, and determined the effect by cell counts. As shown in Fig. 1, the AGS expressed membrane-bound CD40 molecule are on the surface and CD40L have induced cell proliferation.

\section{CD40 ligation stimulated VEGF secretion in AGS cells through PI3K activation}

AGS cells co-cultured with $1 \mu \mathrm{g} / \mathrm{mL}$ sCD40L consistently secreted increasing VEGF secretion, which was first evident at 16 hours lasting up to 72 hour. The highest relative increase compared with control was at 24 hour and this time point was used in further examinations. Next, we pretreated AGS cells with PI3K specific inhibitor LY294002 to prevent activation of PI3K to investigate the mechanism of VEGF production mediated by $\mathrm{SCD} 40 \mathrm{~L}$ since $\mathrm{PI} 3 \mathrm{~K}$ is the major pathway activated by CD40 engagement. Through our initial experiments, we found that $20 \mu \mathrm{M}$ of LY294002 did not show any toxicity on the AGS for experimental period (24 hours) using trypan blue assay. AGS cells were cultured for 24 hours in the presence of $1 \mu \mathrm{g} / \mathrm{mL}$ sCD40L alone or in preincubation with PI3K inhibitor LY294002 $(20 \mu \mathrm{M})$ for one hour. Inhibition of PI3K signaling pathways induced a significant VEGF decrease (Fig. 2). 
VEGFR was essential for the pro-proliferation effect of sCD40L on AGS cells

Because CD40L activated AGS cells growth accompanied by up-regulation of VEGF production, we supposed that VEGF or VGFR signals played an important role in this response. Therefore, AGS cells were cultured in the presence or absence of either anti-VEGF mAb (1 $\mu \mathrm{g} / \mathrm{mL}$ MAB293, isotype IgG as control) or VEGFR inhibitor (8 $\mu \mathrm{M}$ SU5416, $0.2 \%$
DMSO diluent for the SU5416 as control) for one hour before the addition of sCD40L $(1 \mu \mathrm{g} / \mathrm{mL})$. The VEGFR inhibitor SU5416 with a concentration of $8 \mu \mathrm{M}$ lead to a significant decrease in cell survival induced by sCD40L, whereas anti-VEGF mAb did not have the similar effect (Fig. 3). We also found that exogenous VEGF alone had no effect on spontaneous cell growth ( $\mathrm{p}>0.05)$.

A
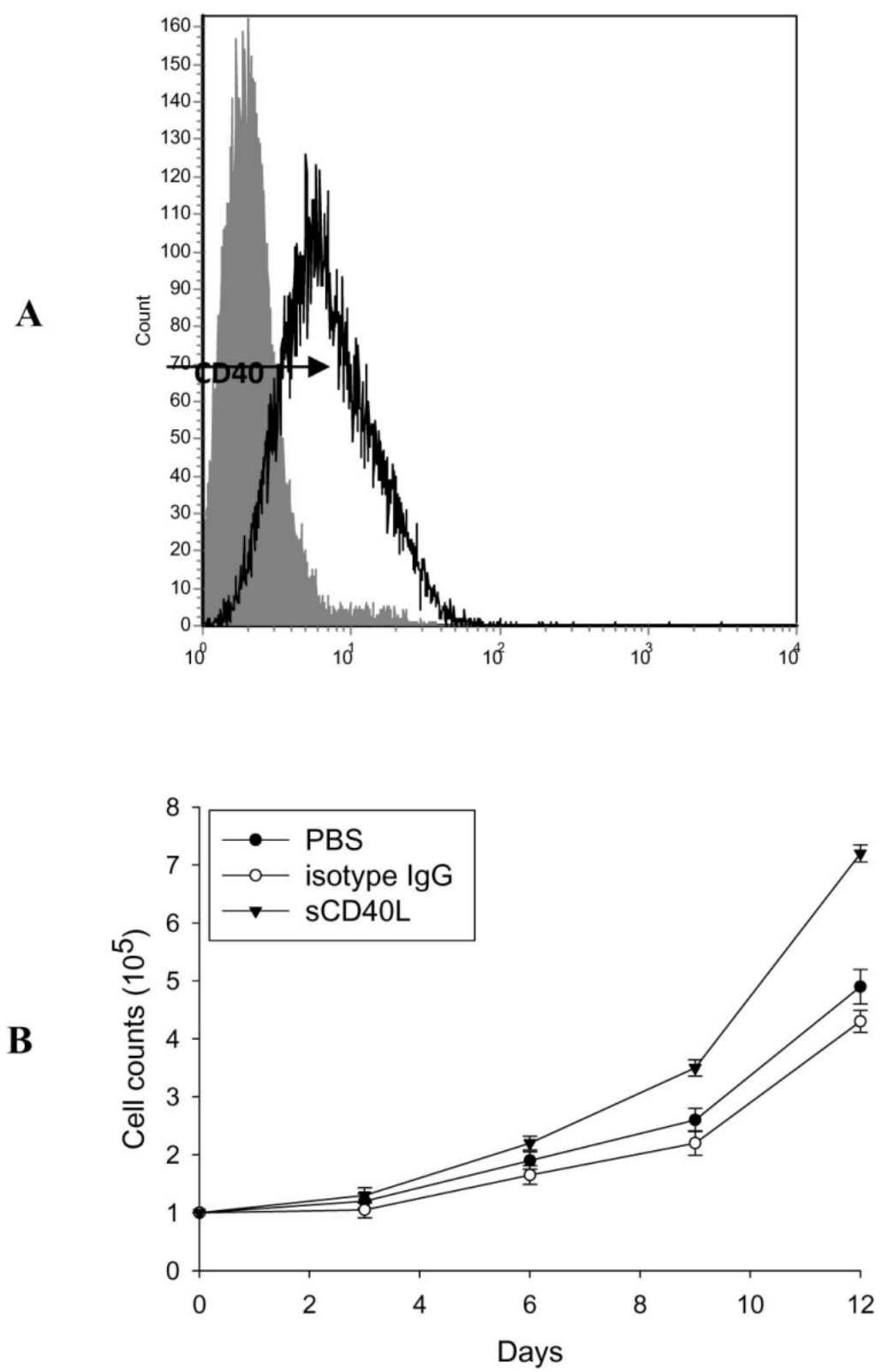

Fig. 1 The effect of sCD40L on the growth of AGS cells. A: Flow cytometric analysis of CD40 expression on AGS cells. The grey filled area indicates isotype control, the black line indicates anti-CD40 antibody. B: $1 \times 10^{5}$ cells per well were cultured with $1 \mu \mathrm{g} / \mathrm{mL}$ sCD40L or PBS as control. The cells were counted under a microscope every three days. The results are presented as the mean of three separate experiments. 


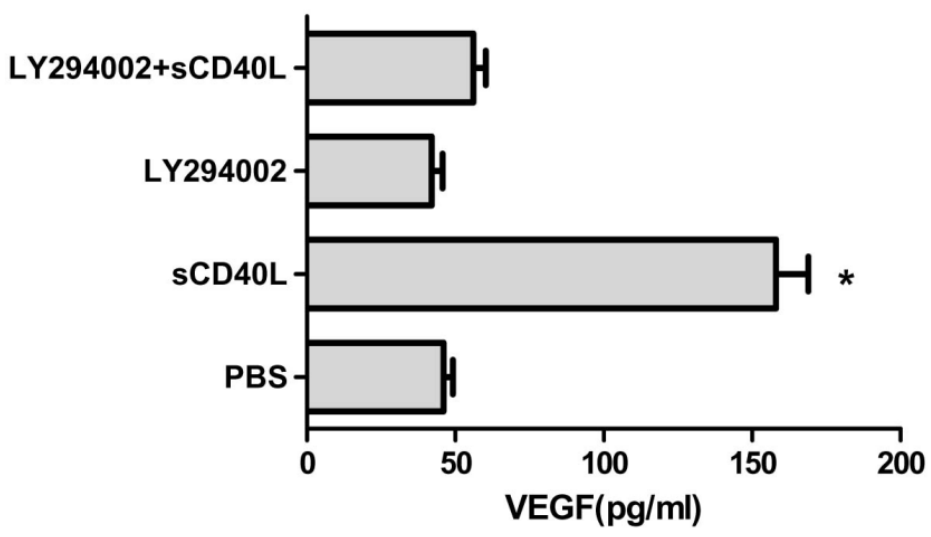

Fig. 2 The effects of CD40 activation on VEGF production in AGS cells. VEGF concentrations were measured by ELISA. Cells were cultured with PBS as negative control. The results are presented as the mean of three separate experiments. *p $<0.05$ compared with either PBS or LY294002+sCD40L groups.

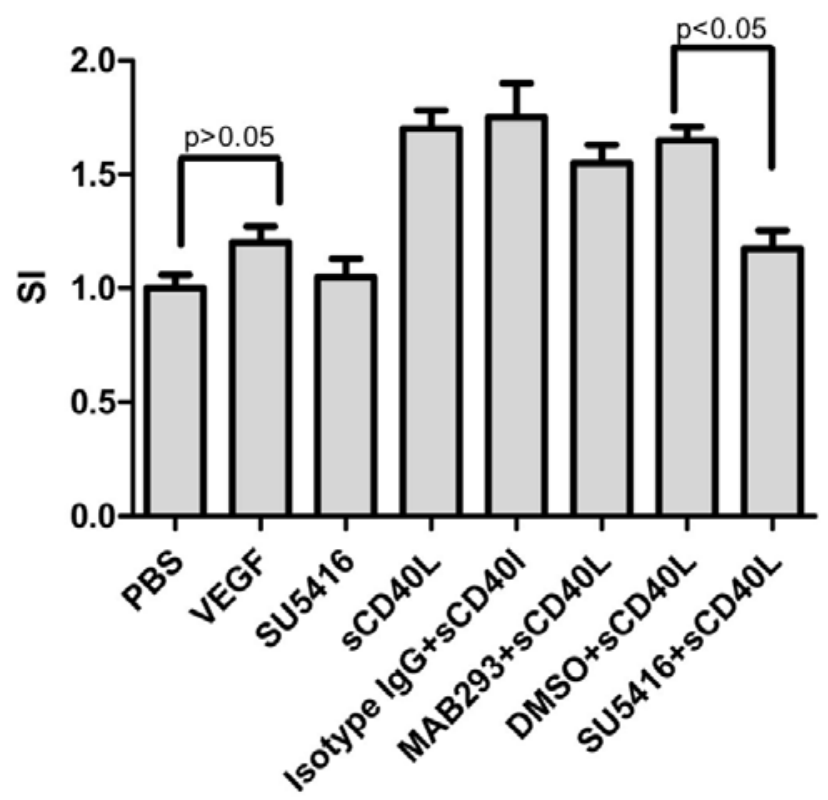

Fig. 3 The role of VEGF in pro-proliferation activity of sCD40L in AGS cells. Cell viability was measured using [3H]-TdR incorporation after four days culture. The results are presented as the mean of three separate experiments.

\section{Discussion}

CD40 lacked intrinsic catalytic kinase activity and transduced signals primarily through trimerization and recruitment of TNF receptor-associated factors (TRAFs) [15]. This resulted in downstream pathways including NF- $\mathrm{KB}$ activation to regulate the growth of cells. Here, we showed that CD40 played an important role in AGS gastric cancer cells and its ligation induced an apparent biological response of proliferation.

Numerous extracellular signals could activate PI3K pathway, which involved cell proliferation, survival, protein synthesis, and tumor growth. VEGF was a key mediator of tumor growth and angiogenesis induced by PI3K-induced signals for tumor growth and angiogenesis, PI3K/AKT signal regulated VEGF expression. In addition, it was critical for regulating VEGF expression and vice versa [16]. The activation of VEGF up-expression was triggered by PI3K, which was associated with CD40 after its ligation by sCD40L. It was reported that the ligation of CD40 on human endothelial cells induced Ras and phosphatidylinositol 3-kinase (PI3K) activation leading to the expression of and the promotion of angiogenesis 
$[17,18]$. In the present study, the presence of PI3K inhibitor, LY294002, could inhibit PI3K activation, VEGF expression, and the pro-proliferation effect in AGS cells. This suggests that the increasing secrete of VEGF partially depended on PI3K activation. This was in accord with previous studies implicating PI3K in the up-regulating of VEGF production in various other cell types [19-22].

CD40-CD40L interactions and the associated expression of VEGF represented a mechanistic link between immunity and the development of angiogenesis [23]. However, we found that blocking autocrine VEGF with an anti-VEGF mAb did not reduce the growth promotion of sCD40L on AGS cells but blocking VEGFR signal pathway significantly did. Such divergent effects between external cytokine blockade and receptor-signaling inhibition had been reported on other cell types [10,24]. We speculated that an internal autocrine loop that could not be inhibited by externally neutralizing antibody might exist in AGS cells. We also observed whether the presence of VEGF alone brought out the same effect as sCD40L. The result showed that exogenous VEGF could not stimulate AGS cells proliferation. This suggested that the pro-proliferation effect of VEGF secreted in response to the engagement of CD40 required both VEGFR and CD40L signals simultaneously.

\section{Conclusion}

Collectively, CD40-CD40L could induce AGS gastric cancer cells proliferation through a VEGFRdependent mechanism with the presence of a VEGF autocrine function specifically linked to the regulation of cells survival.

\section{Acknowledgments}

This work was supported by grants from the Nature Science Fund of China (NSFC) (30901304), and the Project of Changzhou Health Bureau (ZD200902, ZD200907).

The authors have no conflict of interest to declare.

\section{References}

1. Jemal A, Siegel R, Ward E, Hao Y, Xu J, Murray T, Thun MJ. Cancer statistics, 2008. CA Cancer J Clin. 2008; 58 : 71-96.

2. Mizukami Y, Kono K, Kawaguchi Y, Akaike H, Kamimura K, Sugai H, Fujii H. CCL17 and CCL22 chemokines within tumor microenvironment are related to accumulation of Foxp3+ regulatory T cells in gastric cancer. Int J Cancer. 2008; 122:2286-93.

3. Panani AD. Cytogenetic and molecular aspects of gastric cancer: clinical implications. Cancer Lett. 2008; 266:99-115

4. Quezada SA, Jarvinen LZ, Lind EF, Noelle RJ. CD40/ CD154 interactions at the interface of tolerance and immunity. Annu Rev Immunol. 2004; 22:307-328.

5. Vonderheide RH. Prospect of targeting the CD40 pathway for cancer therapy. Clin Cancer Res. 2007; 13: 1083-8.

6. Schonbeck U, Libby P. The CD40/CD154 receptor/ ligand dyad. Cell Mol Life Sci. 2001; 58:4-43.

7. Ochs HD, Hollenbaugh D, Aruffo A. The role of CD40L (gp39)/CD40 in T/B cell interaction and primary immunodeficiency. Semin Immunol. 1994; 6:337-41.

8. Qi CJ, Zheng L, Ma HB, Fei M, Qian KQ, Shen BR, Wu CP, Vihinen M, Zhang XG. A novel mutation in CD40 and its functional characterization. Hum Mutat. 2009; 30:985-94.

9. Murugaiyan G, Martin S, Saha B. CD40-induced countercurrent conduits for tumor escape or elimination? Trends Immunol. 2007; 28:467-73.

10. Farahani M, Treweeke AT, Toh CH, Till KJ, Harris RJ, Cawley JC, Zuzel M, Chen H. Autocrine VEGF mediates the antiapoptotic effect of CD154 on CLL cells. Leukemia. 2005; 19:524-30.

11. Sabel MS, Yamada M, Kawaguchi Y, Chen FA, Takita H, Bankert RB. CD40 expression on human lung cancer correlates with metastatic spread. Cancer Immunol Immunother 2000;49:101-108.

12. Li R, Chen WC, Wang WP, Tian WY, Zhang XG. CD40 signaling activated by agonistic anti-CD40 monoclonal antibody 5C11 has different effects on biological behavior of gastric carcinoma cells. Immunol Lett. 2010; 131:120-5.

13. Kim SW, Lee HS, Yoon SK, Chung WC, Cho YS, Jeong $\mathrm{JJ}$, et al. Expression of CD40 in gastric cancer and its effect on the apoptosis of gastric cancer cells. Korean J Gastroenterol. 2003; 42:274-82.

14. Tomeczkowski J, Beilken A, Frick D, Wieland B, Konig A, Falk MH, et al. Absence of c-kit receptor and absent proliferative response to stem cell factor in childhood Burkitt’s lymphoma cells. Blood. 1995; 86:1469-80.

15. Matsuzawa A, Tseng PH, Vallabhapurapu S, Luo JL, Zhang W, Wang $\mathrm{H}$, et al. Essential cytoplasmic translocation of a cytokine receptor-assembled signaling complex. Science. 2008; 321:663-8.

16. Fang J, Ding M, Yang L, Liu LZ, Jiang BH. PI3K/PTEN/ AKT signaling regulates prostate tumor angiogenesis. 
Cell Signal. 2007; 19:2487-97.

17. Deregibus MC, Buttiglieri S, Russo S, Bussolati B, Camussi G. CD40-dependent activation of phosphatidylinositol 3-kinase/Akt pathway mediates endothelial cell survival and in vitro angiogenesis. J Biol Chem. 2003; 278:18008-14.

18. Shafee N, Kaluz S, Ru N, Stanbridge EJ. PI3K/Akt activity has variable cell-specific effects on expression of HIF target genes, CA9 and VEGF, in human cancer cell lines. Cancer Lett. 2009; 282:109-15.

19. Edelmann J, Klein-Hitpass L, Carpinteiro A, Fuhrer A, Sellmann L, Stilgenbauer S, et al. Bone marrow fibroblasts induce expression of PI3K/NF-kappaB pathway genes and a pro-angiogenic phenotype in CLL cells. Leuk Res. 2008; 32:1565-72.

20. Schnell CR, Stauffer F, Allegrini PR, O’Reilly T, McSheehy PM, Dartois C, et al. Effects of the dual phosphatidylinositol 3-kinase/mammalian target of rapamycin inhibitor NVP-BEZ235 on the tumor vasculature: implications for clinical imaging. Cancer
Res. 2008; 68:6598-607.

21. Kang J, Rychahou PG, Ishola TA, Mourot JM, Evers $\mathrm{BM}$, Chung DH. N-myc is a novel regulator of PI3K-mediated VEGF expression in neuroblastoma. Oncogene. 2008; 27:3999-4007.

22. Yuan TL, Choi HS, Matsui A, Benes C, Lifshits E, Luo J, et al. Class 1A PI3K regulates vessel integrity during development and tumorigenesis. Proc Natl Acad Sci USA. 2008; 105:9739-44.

23. Dormond O, Contreras AG, Meijer E, Datta D, Flynn E, Pal S, et al. CD40-induced signaling in human endothelial cells results in mTORC2- and Aktdependent expression of vascular endothelial growth factor in vitro and in vivo. J Immunol. 2008; 181: 8088-95.

24. Santos SC, Dias S. Internal and external autocrine VEGF/KDR loops regulate survival of subsets of acute leukemia through distinct signaling pathways. Blood. 2004; 103:3883-9. 